\title{
Interference Surge in Full-Duplex Wireless Systems
}

\author{
Ratheesh K. Mungara and Angel Lozano \\ Universitat Pompeu Fabra (UPF) \\ Email: $\{$ ratheesh.mungara, angel.lozano $\} @$ upf.edu
}

\begin{abstract}
Historically unfeasible because of self-interference, full duplexing has now been experimentally demonstrated and is on the verge of commercial feasibility thanks to advances in self-interference cancellation. This will disrupt the interference landscape in wireless networks, bringing about an unprecedented richness whereby every transmitter interferers with every receiver. This paper characterizes the actual increase in system spectral efficiency given all this interference, and in the process it identifies new needs in interference management.
\end{abstract}

Index Terms-Full-duplex, half-duplex, stochastic geometry, interference, ergodic spectral efficiency.

\section{INTRODUCTION}

Full duplexing allows simultaneous transmission and reception on each time-frequency channel, holding the promise of a doubling in spectral efficiency. Historically unfeasible because of self-interference, full duplexing is now becoming possible thanks to advanced combinations of analog and digital cancellation that offer up $110 \mathrm{~dB}$ of transmit-receive isolation [1][4]. By pushing self-interference below the noise level, a neardoubling of the spectral efficiency is theoretically possible for an isolated link. However, this may not extrapolate to links embedded in a network because of the additional interference. The question then arises of whether and when full-duplex is beneficial over half-duplex, and this question is precisely what motivates this paper as well as related works [5]-[7].

\section{NETWORK MODEL}

We consider an interference-limited full-duplex cellular network and conduct the analysis on both forward link (FL) and reverse link (RL). Base stations (BSs) and users have a single antenna and each receiver knows the fading of only its intended signal.

The BS locations $\left\{b_{k}\right\}$ are modeled by a homogeneous Poisson point process (PPP), $\Phi_{\mathrm{b}} \subset \mathbb{R}^{2}$, with density $\lambda_{\mathrm{b}}$. The user locations $\left\{u_{j}\right\}$ are modeled by another independent PPP, $\Phi_{\mathrm{u}} \subset \mathbb{R}^{2}$, with density $\lambda_{\mathrm{u}}=\lambda_{\mathrm{b}}$. In the FL, the receiver under consideration is a user and the transmitter is the closest BS while, in the RL, the receiver under consideration is a BS and the transmitter is the closest user.

To facilitate the readability of the equations, we place $\rightarrow$ and $\leftarrow$ markers atop the FL and RL variables, respectively.

\section{Signal AND PROPAGATION MOdELS}

User antennas are unit-gain while BS antennas have a gain $G_{\mathrm{b}}$. We denote by $P_{\mathrm{b}}$ and $P_{\mathrm{u}}$ the transmit powers of BSs and users, respectively. We consider the generic pathloss model $\beta r^{-\eta}$ where $r$ is the distance and $\eta$ is the exponent while $\beta$ is the intercept, defined as the pathloss at a unit distance.

\section{A. Forward Link}

By Slivnyak's Theorem [8], we consider a receiving user at the origin and focus the analysis on its link, indexed by 0 . This link, whose user and serving BS are respectively located at $u_{0}$ (the origin) and $b_{0}$, serve as the typical link in the network. The user at the origin observes

$$
\vec{y}_{0}=\sqrt{P_{\mathrm{b}} G_{\mathrm{b}} \beta r_{u_{0}, b_{0}}^{-\eta}} h_{u_{0}, b_{0}} s_{b_{0}}+\vec{z}_{0}
$$

whose first term is the signal from the serving $\mathrm{BS}$ at $b_{0}$ while the second term is the interference

$$
\begin{aligned}
\vec{z}_{0}= & \sum_{k=1}^{\infty} \sqrt{P_{\mathrm{b}} G_{\mathrm{b}} \beta r_{u_{0}, b_{k}}^{-\eta}} h_{u_{0}, b_{k}} s_{b_{k}} \\
& +\sum_{j=1}^{\infty} \sqrt{P_{\mathrm{u}} \beta_{\mathrm{u}} r_{u_{0}, u_{j}}^{-\eta_{\mathrm{u}}}} h_{u_{0}, u_{j}} s_{u_{j}}
\end{aligned}
$$

where the first summation spans the interference from other BSs, $\Phi_{\mathrm{b}} \backslash\left\{b_{0}\right\}$, and the second summation spans the interference from other users, $\Phi_{\mathrm{u}} \backslash\left\{u_{0}\right\}$. In turn, $\beta$ and $\beta_{\mathrm{u}}$ are respectively the pathloss intercepts of BS-user and useruser links, $\eta$ and $\eta_{\mathrm{u}}$ are the corresponding pathloss exponents, $r_{u_{0}, b_{0}}$ is the distance from $b_{0}$ to $u_{0}$, and $h_{u_{0}, b_{0}}$ is the corresponding fading coefficient. The fading coefficients are complex Gaussian with zero mean and unit variance, i.e., $h_{u_{0}, b_{k}} \sim \mathcal{N}_{\mathbb{C}}(0,1)$ and $h_{u_{0}, u_{j}} \sim \mathcal{N}_{\mathbb{C}}(0,1)$. Meanwhile, $s_{b_{k}} \sim \mathcal{N}_{\mathbb{C}}(0,1)$ and $s_{u_{j}} \sim \mathcal{N}_{\mathbb{C}}(0,1)$ are the data symbols transmitted by the BS at $b_{k}$ and by the user at $u_{j}$, respectively.

Without loss of generality, BS and user locations are indexed in order of increasing distance, i.e., $r_{u_{0}, b_{k}}<r_{u_{0}, b_{k+1}}$ and $r_{u_{0}, u_{j}}<r_{u_{0}, u_{j+1}}$.

\section{B. Reverse Link}

We adopt the two-slope model for BS-BS pathloss. Denoting by $R_{\mathrm{c}}=4 \mathrm{~h}_{\mathrm{b}}^{2} / \lambda$ the critical distance with $\mathrm{h}_{\mathrm{b}}$ the BS antenna height and $\lambda$ the wavelength, the pathloss for a given link distance $r_{b_{0}, b_{k}}$ is

$$
\begin{cases}\beta_{\mathrm{b}} r_{b_{0}, b_{k}}^{-\eta_{\mathrm{b}}} & r_{b_{0}, b_{k}} \leq R_{\mathrm{c}} \\ \beta_{\mathrm{B}} r_{b_{0}, b_{k}}^{-\eta_{\mathrm{B}}} & r_{b_{0}, b_{k}}>R_{\mathrm{c}}\end{cases}
$$

where $\eta_{\mathrm{b}}$ and $\beta_{\mathrm{b}}$ are respectively the pathloss exponent and intercept of BS-BS links satisfying $r_{b_{0}, b_{k}} \leq R_{\mathrm{c}}$ while $\eta_{\mathrm{B}}$ and $\beta_{\mathrm{B}}$ are the pathloss exponent and intercept of BS-BS links satisfying $r_{b_{0}, b_{k}}>R_{\mathrm{c}}$. To analyze the RL, we shift the origin to the $\mathrm{BS}$ of interest, which observes

$$
\overleftarrow{y}_{0}=\sqrt{P_{\mathrm{u}} G_{\mathrm{b}} \beta r_{b_{0}, u_{0}}^{-\eta}} h_{b_{0}, u_{0}} s_{u_{0}}+\overleftarrow{z}_{0}
$$


whose first term is the signal from its intended user while the second term is the aggregate interference

$$
\begin{aligned}
\overleftarrow{z}_{0}= & \sum_{j=1}^{\infty} \sqrt{P_{\mathrm{u}} G_{\mathrm{b}} \beta r_{b_{0}, u_{j}}^{-\eta}} h_{b_{0}, u_{j}} s_{u_{j}} \\
& +\sum_{k \in \mathcal{K}} \sqrt{P_{\mathrm{b}} G_{\mathrm{b}}^{2} \beta_{\mathrm{b}} r_{b_{0}, b_{k}}^{-\eta_{\mathrm{b}}}} h_{b_{0}, b_{k}} s_{b_{k}} \\
& +\sum_{k \notin \mathcal{K}} \sqrt{P_{\mathrm{b}} G_{\mathrm{b}}^{2} \beta_{\mathrm{B}} r_{b_{0}, b_{k}}^{-\eta_{\mathrm{B}}}} h_{b_{0}, b_{k}} s_{b_{k}}
\end{aligned}
$$

where the first summation spans the interference from other users, $\Phi_{\mathrm{u}} \backslash\left\{u_{0}\right\}$, and the second and third summations span the interference from other BSs, $\Phi_{\mathrm{b}} \backslash\left\{b_{0}\right\}$. In (5), $\mathcal{K}=\{k$ : $\left.r_{b_{0}, b_{k}} \leq R_{c}\right\}$ and $h_{b_{0}, b_{k}} \sim \mathcal{N}_{\mathbb{C}}(0,1)$ is the fading coefficient from the BS at $b_{k}$ to the BS at $b_{0}$.

\section{INTERFERENCE MODELING}

We follow the approach in [9] to model the interference terms $\vec{z}_{0}$ and $\overleftarrow{z}_{0}$. The short-term (local-average) distributions of $\vec{z}_{0}$ and $\overleftarrow{z}_{0}$ are modeled as zero-mean complex Gaussian with respective matched conditional covariances $\mathbb{E}\left[\left|\vec{z}_{0}\right|^{2} \mid\left\{r_{u_{0}, b_{k}}, r_{u_{0}, u_{j}}\right\}\right]$ and $\mathbb{E}\left[\left|\overleftarrow{z}_{0}\right|^{2} \mid\left\{r_{b_{0}, u_{j}}, r_{b_{0}, b_{k}}\right\}\right]$ where the expectations are over the data and fading distributions.

Recalling (2), the conditional covariance of $\vec{z}_{0}$ for given interferer locations equals

$\mathbb{E}\left[\left|\vec{z}_{0}\right|^{2} \mid\left\{r_{u_{0}, b_{k}}, r_{u_{0}, u_{j}}\right\}\right]=P_{\mathrm{b}} G_{\mathrm{b}} \beta \sum_{k=1}^{\infty} r_{u_{0}, b_{k}}^{-\eta}+P_{\mathrm{u}} \beta_{\mathrm{u}} \sum_{j=1}^{\infty} r_{u_{0}, u_{j}}^{-\eta_{\mathrm{u}}}$

while, recalling (5), its RL counterpart equals

$$
\begin{gathered}
\mathbb{E}\left[\left|\overleftarrow{z}_{0}\right|^{2} \mid\left\{r_{b_{0}, u_{j}}, r_{b_{0}, b_{k}}\right\}\right]=P_{\mathrm{u}} G_{\mathrm{b}} \beta \sum_{j=1}^{\infty} r_{b_{0}, u_{j}}^{-\eta} \\
+P_{\mathrm{b}} G_{\mathrm{b}}^{2} \beta_{\mathrm{b}} \sum_{k \in \mathcal{K}} r_{b_{0}, b_{k}}^{-\eta_{\mathrm{b}}}+P_{\mathrm{b}} G_{\mathrm{b}}^{2} \beta_{\mathrm{B}} \sum_{k \notin \mathcal{K}} r_{b_{0}, b_{k}}^{-\eta_{\mathrm{B}}} .
\end{gathered}
$$

\section{SIR DISTRIBUTIONS}

\section{A. Forward Link}

From (1) and (6), the instantaneous SIR (signal-tointerference ratio) of the typical user in the FL is

$$
\begin{aligned}
\overrightarrow{\operatorname{SIR}}_{0} & =\frac{P_{\mathrm{b}} G_{\mathrm{b}} \beta r_{u_{0}, b_{0}}^{-\eta} \mathbb{E}\left[\left|h_{u_{0}, b_{0}} s_{b_{0}}\right|^{2} \mid h_{u_{0}, b_{0}}\right]}{\mathbb{E}\left[\left|\vec{z}_{0}\right|^{2} \mid\left\{r_{u_{0}, b_{k}}, r_{u_{0}, u_{j}}\right\}\right]} \\
& =\vec{\rho}_{0}\left|h_{u_{0}, b_{0}}\right|^{2}
\end{aligned}
$$

where

$$
\vec{\rho}_{0}=\frac{r_{u_{0}, b_{0}}^{-\eta}}{\sum_{j=1}^{\infty} r_{u_{0}, u_{j}}^{-\eta_{\mathrm{u}}}+\mu_{\mathrm{u}} \sum_{k=1}^{\infty} r_{u_{0}, b_{k}}^{-\eta}}
$$

is the local-average SIR at the typical user in the FL with

$$
\mu_{\mathrm{u}}=\frac{P_{\mathrm{u}} \beta_{\mathrm{u}}}{P_{\mathrm{b}} G_{\mathrm{b}} \beta}
$$

1) Local-Average SIR Distribution: The spatial distribution of the transmitter locations induces a distribution of its own for $\vec{\rho}_{0}$, i.e., a long-term distribution for the local-average SIR, which is derived next. As the distribution of the local-average interference generally does not admit a closed-form, certain approximate characterizations have been proposed. Here, we adapt the approach in [10], which is based on the Euler series expansion of the numerical inversion of the Laplace transform of the interference distribution. We introduce $\Re(\cdot), \Gamma(\cdot)$ and $\bar{\Gamma}(\cdot, \cdot)$ to denote the real part, the Gamma function and the lower incomplete Gamma function.

Proposition 1. For a given $r_{u_{0}, b_{0}}$, the CDF of $\vec{\rho}_{0}$ is

$$
\begin{gathered}
F_{\vec{\rho}_{0} \mid r_{u_{0}, b_{0}}}(\gamma) \approx 1-\frac{\gamma 10^{\frac{\zeta}{2}}}{2^{L}} \sum_{\ell=0}^{L}\left(\begin{array}{c}
L \\
\ell
\end{array}\right) \sum_{m=0}^{M+\ell} \frac{(-1)^{m}}{D_{m}} \\
. \Re\left\{\frac { 1 } { t } \operatorname { e x p } \left(\pi \lambda_{\mathrm{b}} r_{u_{0}, b_{0}}^{2}+\frac{2 \pi \lambda_{\mathrm{b}}}{\eta} r_{u_{0}, b_{0}}^{2} t^{\frac{2}{\eta}} \bar{\Gamma}\left(-\frac{2}{\eta}, t\right)\right.\right. \\
\left.\left.-\pi \lambda_{\mathrm{b}}\left(r_{u_{0}, b_{0}}^{\eta} \mu_{\mathrm{u}} t\right)^{\frac{2}{\eta_{\mathrm{u}}}} \Gamma\left(1-\frac{2}{\eta_{\mathrm{u}}}\right)\right)\right\}
\end{gathered}
$$

where $t=\frac{\left(\zeta \log _{e} 10+\mathrm{i} 2 \pi m\right) \gamma}{2}$ while $D_{0}=2$ and $D_{m}=1$ for $m>1$. The parameter $\zeta$, through $L$ and $M$, controls the absolute difference between the exact distribution and the approximation: an accuracy of $10^{-\zeta}$ requires, respectively, $L=\lceil 1.243 \zeta-1\rceil$ and $M=\lceil 1.476 \zeta\rceil[10]$.

Proof. See [11].

Eq. (12) can be further averaged over $r_{u_{0}, b_{0}}$ via

$$
f_{r_{u_{0}, b_{0}}}(r)=2 \pi \lambda_{\mathrm{b}} r e^{-\pi \lambda_{\mathrm{b}} r^{2}}
$$

to obtain the unconditional CDF of the local-average SIR,

$$
F_{\vec{\rho}_{0}}(\gamma)=\int_{0}^{\infty} F_{\vec{\rho}_{0} \mid r_{u_{0}, b_{0}}}(\gamma) f_{r_{u_{0}, b_{0}}}(r) \mathrm{d} r
$$

TABLE I: Microcell Network Settings [12, Scenario 2]

\begin{tabular}{|c|c||c|c|}
\hline Parameter & Value & Parameter & Value \\
\hline \hline$P_{\mathrm{b}}$ & $24 \mathrm{dBm}$ & $\eta$ & 3.75 \\
\hline$P_{\mathrm{u}}$ & $23 \mathrm{dBm}$ & $\eta_{\mathrm{b}}$ & 2 \\
\hline$G_{\mathrm{b}}$ & $5 \mathrm{dBi}$ & $\eta_{\mathrm{B}}$ & 4 \\
\hline$\beta$ & $-32.9 \mathrm{~dB}$ & $\eta_{\mathrm{u}}$ & 4 \\
\hline$\beta_{\mathrm{b}}$ & $-38.45 \mathrm{~dB}$ & $\mathrm{~h}_{\mathrm{b}}$ & $4 \mathrm{~m}$ \\
\hline$\beta_{\mathrm{B}}$ & $-49.36 \mathrm{~dB}$ & $\lambda$ & $15 \mathrm{~cm}$ \\
\hline$\beta_{\mathrm{u}}$ & $-55.78 \mathrm{~dB}$ & $\lambda_{\mathrm{b}}$ & $7.95 \mathrm{BSs} / \mathrm{km}^{2}$ \\
\hline
\end{tabular}

Example 1. Consider a network with $\lambda_{\mathrm{b}}=7.95 \mathrm{BSs} / \mathrm{km}^{2}$, which amounts to an average of one BS per circular cell of radius $200 \mathrm{~m}$ and with typical values for the powers and the pathloss exponents (cf. Table I). Shown in Fig. 1 is a comparison of $F_{\vec{\rho}_{0}} \mid r_{u_{0}, b_{0}}(\cdot)$ and $F_{\vec{\rho}_{0}}(\cdot)$ in (12) and (14), with $\zeta=4$, $L=5$ and $M=6$, against their Monte-Carlo counterparts. The conditional CDF corresponds to $r_{u_{0}, b_{0}}=150 \mathrm{~m}$.

An excellent match is observed, supporting the validity of the Euler series expansion of the inverse Laplace transform. 


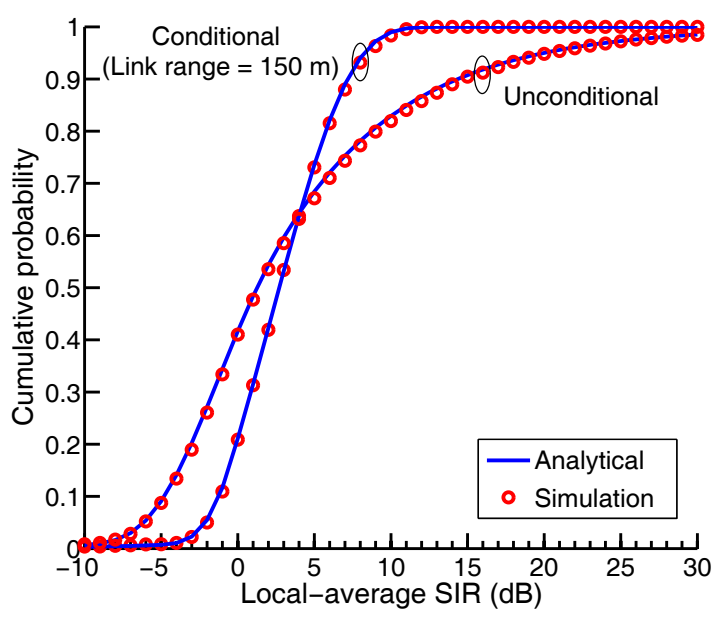

Fig. 1: CDF of FL local-average SIR in a full-duplex microcell network.

2) Instantaneous SIR Distribution: Given $\left\{r_{u_{0}, b_{k}}\right\}$ and $\left\{r_{u_{0}, u_{j}}\right\}$, the value of $\vec{\rho}_{0}$ becomes determined and, from (9),

$$
F_{\overrightarrow{\mathrm{SIR}}_{0} \mid \vec{\rho}_{0}}(\gamma)=1-e^{-\gamma / \vec{\rho}_{0}}
$$

\section{B. Reverse Link}

From (4), the RL instantaneous SIR at the receiving BS is

$$
\overleftarrow{S I R}_{0}=\overleftarrow{\rho}_{0}\left|h_{b_{0}, u_{0}}\right|^{2}
$$

where

$$
{\stackrel{\leftarrow}{\rho_{0}}}_{0}=\frac{r_{b_{0}, u_{0}}^{-\eta}}{\sum_{j=1}^{\infty} r_{b_{0}, u_{j}}^{-\eta}+\mu_{\mathrm{b}} \sum_{k \in \mathcal{K}} r_{b_{0}, b_{k}}^{-\eta_{\mathrm{b}}}+\mu_{\mathrm{B}} \sum_{k \notin \mathcal{K}} r_{b_{0}, b_{k}}^{-\eta_{\mathrm{B}}}}
$$

is the RL local-average SIR at the typical BS with

$$
\begin{aligned}
\mu_{\mathrm{b}} & =\frac{P_{\mathrm{b}} G_{\mathrm{b}} \beta_{\mathrm{b}}}{P_{\mathrm{u}} \beta} \\
\mu_{\mathrm{B}} & =\frac{P_{\mathrm{b}} G_{\mathrm{b}} \beta_{\mathrm{B}}}{P_{\mathrm{u}} \beta} .
\end{aligned}
$$

1) Local-Average SIR Distribution: Noting that BSs are not arbitrarily close in actual deployments, we introduce a parameter $0<\kappa \leq 1$ such that $r_{b_{0}, b_{1}}>\kappa R_{\mathrm{c}}$. We derive the CDF of $\overleftarrow{\rho}_{0}$ in terms of $\kappa$, thereby parameterizing the distribution by the guaranteed distance to the strongest interfering BS.
Proposition 2. For given $r_{u_{0}, b_{0}}$, the CDF of $\overleftarrow{\rho}_{0}$ is

$$
\begin{aligned}
& F_{\bar{\rho}_{0} \mid r_{b_{0}, u_{0}}}(\gamma) \approx 1-\frac{\gamma 10^{\frac{\zeta}{2}}}{2^{L}} \sum_{\ell=0}^{L}\left(\begin{array}{l}
L \\
\ell
\end{array}\right) \sum_{m=0}^{M+\ell} \frac{(-1)^{m}}{D_{m}} \\
& \text {. } \Re\left\{\frac{1}{t} e^{\pi \lambda_{\mathrm{b}} r_{b_{0}, u_{0}}^{2}+\pi \lambda_{\mathrm{b}} \kappa^{2} R_{\mathrm{c}}^{2}+\frac{2 \pi \lambda_{\mathrm{b}}}{\eta} r_{b_{0}, u_{0}}^{2} t^{\frac{2}{\eta}} \bar{\Gamma}\left(-\frac{2}{\eta}, t\right)}\right. \\
& \cdot e^{\frac{2 \pi \lambda_{\mathrm{b}} R_{\mathrm{c}}^{2}}{\eta_{\mathrm{b}}}}\left(E_{\frac{\eta_{\mathrm{b}}+2}{\eta_{\mathrm{b}}}}\left(\frac{r_{b_{0}, u_{0}}^{\eta}}{R_{\mathrm{c}}^{\eta_{\mathrm{b}}}} \mu_{\mathrm{b}} t\right)-\kappa^{2} E_{\frac{\eta_{\mathrm{b}}+2}{\eta_{\mathrm{b}}}}\left(\frac{r_{b_{0}, u_{0}}^{\eta}}{\left(\kappa R_{\mathrm{c}}\right)^{\eta_{\mathrm{b}}}} \mu_{\mathrm{b}} t\right)\right) \\
& \left.\cdot e^{\frac{2 \pi \lambda_{\mathrm{b}}}{\eta_{\mathrm{B}}}\left(r_{b_{0}, u_{0}}^{\eta} \mu_{\mathrm{B}} t\right)^{\frac{2}{\eta_{\mathrm{B}}}} \bar{\Gamma}\left(-\frac{2}{\eta_{\mathrm{B}}}, \frac{r_{b_{0}, u_{0}}^{\eta}}{R_{\mathrm{C}}^{\eta_{\mathrm{B}}}} \mu_{\mathrm{B}} t\right)}\right\}
\end{aligned}
$$

where $t$ and $D_{m}$ are as in Proposition 1 and $E_{n}(\zeta)=$ $\int_{1}^{\infty} \frac{e^{-\zeta t}}{t^{n}} \mathrm{~d} t$ is an exponential integral.

Proof. See [11].

The unconditional CDF of local-average SIR can be obtained as

$$
F_{\bar{\rho}_{0}}(\gamma)=\int_{0}^{\infty} F_{\bar{\rho}_{0} \mid r_{b_{0}, u_{0}}}(\gamma) f_{r_{b_{0}, u_{0}}}(r) \mathrm{d} r
$$

where $f_{r_{b_{0}, u_{0}}}(\cdot)$ was given in (13).

2) Instantaneous SIR Distribution: Given $\left\{r_{b_{0}, u_{j}}\right\}$ and $\left\{r_{b_{0}, b_{k}}\right\}, \overleftarrow{\rho}_{0}$ in (17) becomes determined and, from (16),

$$
F_{\overleftarrow{S I R}_{0} \mid \overleftarrow{\rho}_{0}}(\gamma)=1-e^{-\gamma / \overleftarrow{\rho}_{0}}
$$

\section{Spectral EFFICIENCY}

\section{A. Forward Link}

1) Specific Network Geometries: For given $\vec{\rho}_{0}$, the ergodic link spectral efficiency in the FL is

$$
\begin{aligned}
\vec{C}\left(\vec{\rho}_{0}\right) & =\int_{0}^{\infty} \log _{2}(1+\gamma) \mathrm{d} F_{\overrightarrow{S I R}_{0} \mid \vec{\rho}_{0}}(\gamma) \\
& =e^{1 / \vec{\rho}_{0}} E_{1}\left(\frac{1}{\vec{\rho}_{0}}\right) \log _{2} e
\end{aligned}
$$

and its CDF equals

$$
F_{\vec{C}}(\gamma)=\mathbb{P}\left[e^{1 / \vec{\rho}_{0}} E_{1}\left(\frac{1}{\vec{\rho}_{0}}\right) \log _{2} e<\gamma\right]
$$

At this point, we invoke

$$
e^{\nu} E_{1}(\nu) \log _{2} e \approx 1.4 \log _{e}\left(1+\frac{0.82}{\nu}\right)
$$

and approximate (25) as

$$
F_{\vec{C}}(\gamma) \approx F_{\vec{\rho}_{0}}\left(\frac{e^{\frac{\gamma}{1.4}}-1}{0.82}\right)
$$

which is validated in the following example.

Example 2. Reconsider Example 1. In Fig. 2, the approximated CDFs $F_{\vec{C} \mid r_{u_{0}, b_{0}}}(\gamma)$ and $F_{\vec{C}}(\gamma)$ are contrasted against the ones obtained through Monte-Carlo.

Very good agreements are observed, supporting (27). 


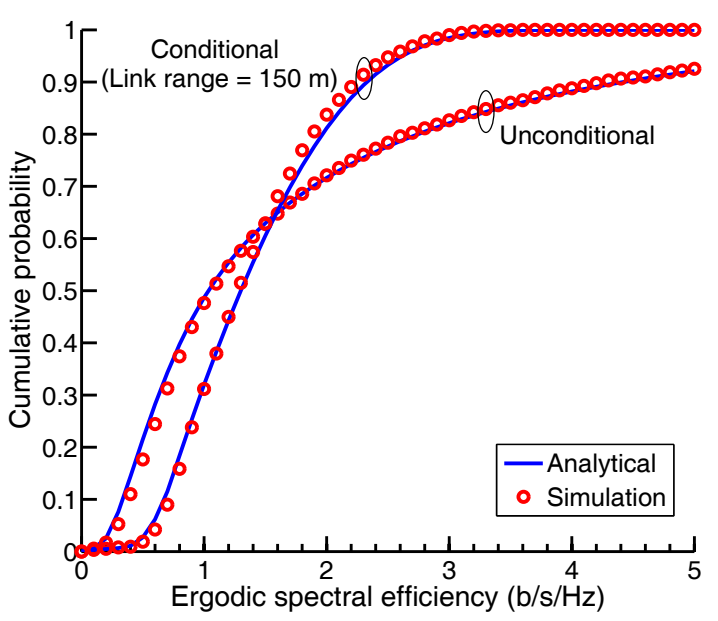

Fig. 2: CDF of FL ergodic spectral efficiency.

2) Average Network Geometry: Next, we average the link spectral efficiency over all possible geometries.

Proposition 3. The average FL spectral efficiency of a fullduplex network is

$$
\begin{gathered}
\overrightarrow{\vec{C}}=\int_{0}^{\infty} \frac{\log _{2} e}{\gamma+1} \int_{r>0}^{\infty} 2 \pi \lambda_{\mathrm{b}} r e^{\frac{2 \pi \lambda_{\mathrm{b}}}{\eta} \gamma^{\frac{2}{\eta}} r^{2} \bar{\Gamma}\left(-\frac{2}{\eta}, \gamma\right)} \\
\cdot e^{-\pi \lambda_{\mathrm{b}}\left(r^{\eta} \mu_{\mathrm{u}} \gamma\right)^{\frac{2}{\eta_{\mathrm{u}}}} \Gamma\left(1-\frac{2}{\eta_{\mathrm{u}}}\right)} \mathrm{d} r \mathrm{~d} \gamma
\end{gathered}
$$

Proof. See [11].

Example 3. For the microcell parameters in Table I, the average spectral efficiency computed via (28) is $1.78 \mathrm{~b} / \mathrm{s} / \mathrm{Hz}$ while its simulated counterpart is $1.82 \mathrm{~b} / \mathrm{s} / \mathrm{Hz}$. The simulated result corresponds to the exact mutual information under the non-Gaussian interference in (2), evaluated through MonteCarlo and averaged over the fading and interference locations.

\section{B. Reverse Link}

1) Specific Network Geometries: For given $\overleftarrow{\rho}_{0}$,

$$
\overleftarrow{C}\left(\overleftarrow{\rho}_{0}\right)=e^{1 / \bar{\rho}_{0}} E_{1}\left(\frac{1}{\overleftarrow{\rho}_{0}}\right) \log _{2} e
$$

with $\mathrm{CDF}$

$$
F_{\bar{C}}(\gamma) \approx F_{\bar{\rho}_{0}}\left(\frac{e^{\frac{\gamma}{1.4}}-1}{0.82}\right) .
$$

2) Average Network Geometry: By leveraging the derivation of its FL counterpart, we obtain the RL average spectral efficiency as follows.

Proposition 4. Conditioned on $r_{b_{0}, b_{1}}>\kappa R_{c}$, the average $R L$ spectral efficiency of a full-duplex network is

$$
\begin{aligned}
& \stackrel{\bar{C}}{=} \int_{0}^{\infty} \frac{\log _{2} e}{\gamma+1} \int_{0}^{\infty} 2 \pi \lambda_{\mathrm{b}} r e^{\pi \lambda_{\mathrm{b}} \kappa^{2} R_{\mathrm{c}}^{2}+\frac{2 \pi \lambda_{\mathrm{b}} r^{2} \gamma^{\frac{2}{\eta}}}{\eta}} \bar{\Gamma}\left(\frac{-2}{\eta}, \gamma\right) \\
& \cdot e^{\frac{2 \pi \lambda_{\mathrm{b}} R_{c}^{2}}{\eta_{\mathrm{b}}}}\left(E_{\frac{\eta_{\mathrm{b}}+2}{\eta_{\mathrm{b}}}}\left(\frac{r^{\eta}}{R_{\mathrm{c}} \eta_{\mathrm{b}}} \mu_{\mathrm{b}} \gamma\right)-\kappa^{2} E_{\frac{\eta_{\mathrm{b}}+2}{\eta_{\mathrm{b}}}}\left(\frac{r^{\eta}}{\left(\kappa R_{\mathrm{c}}\right)^{\eta} \eta_{\mathrm{b}}} \mu_{\mathrm{b}} \gamma\right)\right) \\
& \cdot e^{\frac{2 \pi \lambda_{\mathrm{b}}}{\eta_{\mathrm{B}}}\left(r^{\eta} \mu_{\mathrm{B}} \gamma\right)^{\frac{2}{\eta_{\mathrm{B}}}} \bar{\Gamma}\left(-\frac{2}{\eta_{\mathrm{B}}}, \frac{r^{\eta}}{R_{\mathrm{C}} \mathrm{B}^{\mathrm{B}}} \mu_{\mathrm{B}} \gamma\right)} \mathrm{d} r \mathrm{~d} \gamma \text {. }
\end{aligned}
$$

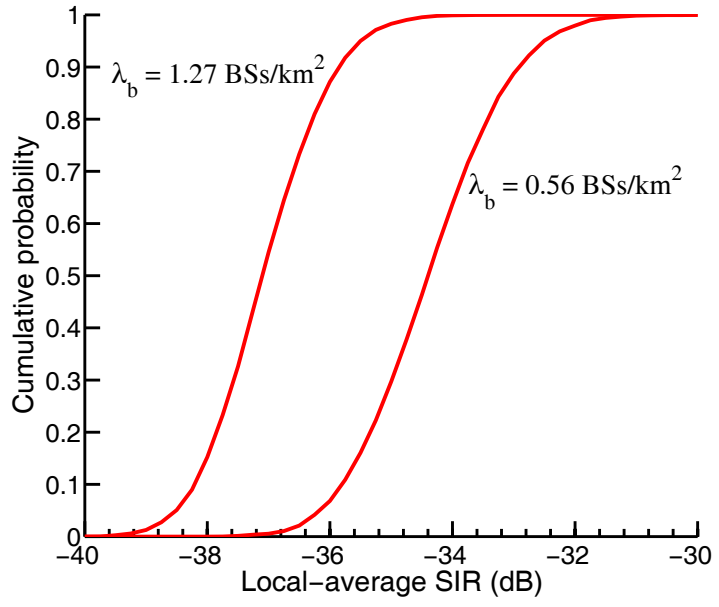

Fig. 3: CDF of RL local-average SIR as function of $\lambda_{\mathrm{b}}$, for a full-duplex macrocell network with $r_{b_{0}, u_{0}}=100 \mathrm{~m}$ and $\kappa=0.1$ (equivalently, $r_{b_{0}, b_{1}}>1060 \mathrm{~m}$ ).

\section{ViI. Performance of Full-Duplex Communication}

\section{A. Half-Duplex Baseline}

From (24), (14) and (28), we can recover the FL counterparts for half-duplex as

$$
\begin{aligned}
\vec{C}^{\mathrm{HD}} & =\frac{1}{2} \lim _{\mu_{\mathrm{u}} \rightarrow 0} \vec{C}\left(\vec{\rho}_{0}\right) \\
F_{\vec{C}^{\mathrm{HD}}}(\gamma) & \approx \lim _{\mu_{\mathrm{u}} \rightarrow 0} F_{\vec{\rho}_{0}}\left(\frac{e^{\frac{\gamma}{0.7}}-1}{0.82}\right) \\
\overrightarrow{\vec{C}}^{\mathrm{HD}} & =\frac{1}{2} \lim _{\mu_{\mathrm{u}} \rightarrow 0} \overrightarrow{\vec{C}}\left(\vec{\rho}_{0}\right)
\end{aligned}
$$

where $\mu_{\mathrm{u}} \rightarrow 0$ turns off the FL user transmissions while the factor $1 / 2$ accounts for the separate forward/reverse channels.

Similarly, from (29), (21) and (31), we can recover the RL counterparts for half-duplex by setting $\mu_{\mathrm{b}} \rightarrow 0, \mu_{\mathrm{B}} \rightarrow 0$ and $\kappa \rightarrow 0$, i.e., by turning off the RL BS transmissions.

\section{B. Performance Evaluation}

Consider a macrocellular network with $P_{\mathrm{b}}=46 \mathrm{dBm}, P_{\mathrm{u}}=$ $23 \mathrm{dBm}, \beta=-15.3 \mathrm{~dB}, \eta=3.75, \beta_{\mathrm{b}}=-38.45 \mathrm{~dB}, \eta_{\mathrm{b}}=2$, $\beta_{\mathrm{B}}=1.0439 \mathrm{~dB}, \eta_{\mathrm{B}}=4$ and $R_{\mathrm{c}}=10.6 \mathrm{~km} \mathrm{[12].} \mathrm{BS} \mathrm{densities}$ $\lambda_{\mathrm{b}}=1.27 \mathrm{BSs} / \mathrm{km}^{2}$ and $\lambda_{\mathrm{b}}=0.56 \mathrm{BSs} / \mathrm{km}^{2}$ are considered, respectively amounting to an average of one BS per circular cell of radii $500 \mathrm{~m}$ and $750 \mathrm{~m}$. The intended link distance is $r_{b_{0}, u_{0}}=100 \mathrm{~m}$ while $\kappa=0.1$ (equivalently, $r_{b_{0}, b_{1}}>1060$ $\mathrm{m})$. We consider idealized sector antennas at the BSs with gain pattern

$$
G_{\mathrm{b}}=\left\{\begin{array}{ccc}
15 \mathrm{dBi} & \text { with probability } & 1 / 3 \\
-5 \mathrm{dBi} & \text { with probability } & 2 / 3
\end{array}\right.
$$

Fig. 3 shows, as a function of $\lambda_{\mathrm{b}}$, the $\mathrm{CDF}$ of $\hat{\rho}_{0}$ obtained via Monte-Carlo. The excessive interference among macro BSs, due to the low BS-BS pathloss for link ranges below $R_{\mathrm{c}}$, yields SIRs that are simply too low for viable full-duplex operation. 


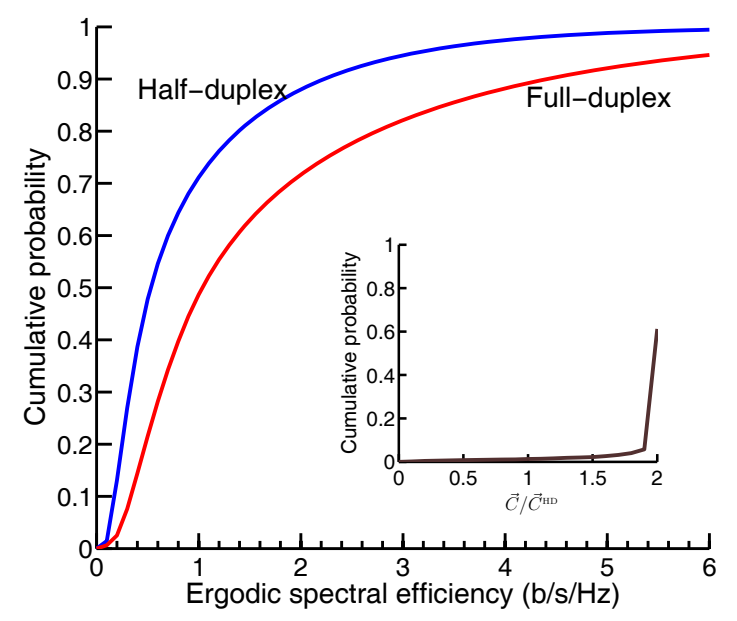

Fig. 4: Main plot: CDF of FL ergodic spectral efficiency for a microcell network with half-duplex or full-duplex. Inset: CDF of the ratio of the two spectral efficiencies.

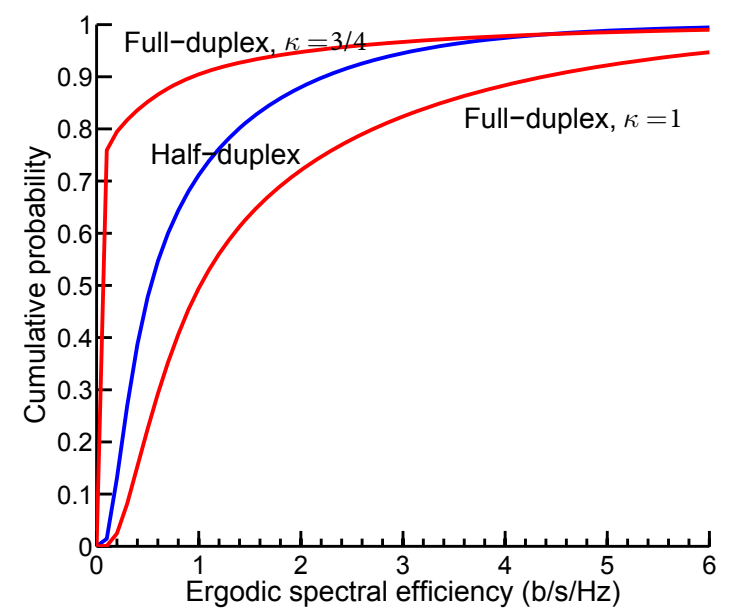

Fig. 5: CDF of RL ergodic spectral efficiency for a microcell network with half-duplex and full-duplex.

Having exemplified how, without additional dedicated interference management, full-duplex is not feasible in macrocell RLs, we henceforth focus on microcells (cf. Table I). Fig. 4 compares the FL spectral efficiency CDFs of full-duplex and half-duplex (cf. (27) and (33)). Full-duplex is superior to half-duplex in a vast majority of network situations and, as illustrated in the inset of Fig. 4, it achieves a spectral efficiency gain factor of 1.9 in $80 \%$ of situations. Then, Fig. 5 presents the same comparison for the RL, with two distinct values for $\kappa$. When $\kappa=3 / 4$, i.e., when the first interfering BS is within a critical distance of the receiving $\mathrm{BS}$, full-duplex is markedly inferior to half-duplex. However, for $\kappa=1$, full-duplex is already uniformly superior to half-duplex, pointing to the need for a careful planning in full-duplex deployments.

Finally, we quantify the average benefits of full-duplex, still for the microcell settings in Table I. The FL average spectral efficiencies (cf. (28) and (34)) are $1.78 \mathrm{~b} / \mathrm{s} / \mathrm{Hz}$ and

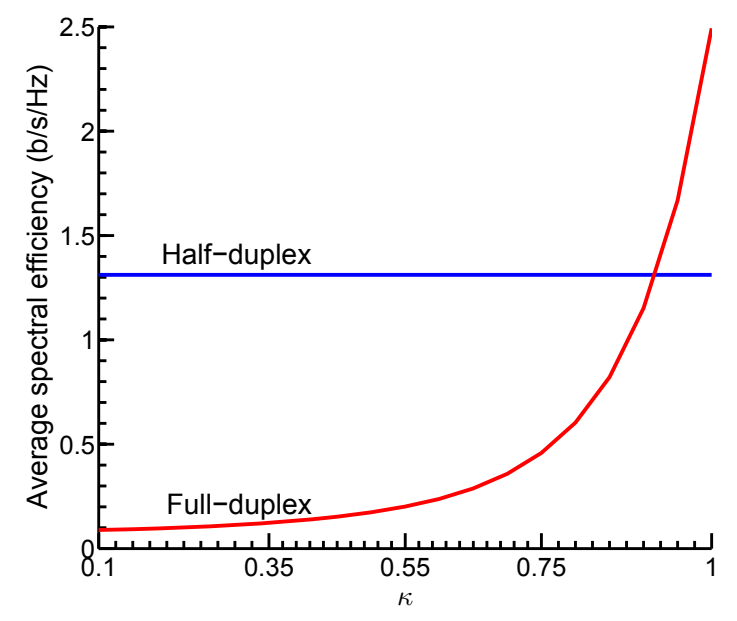

Fig. 6: RL average spectral efficiency for a microcell network with half-duplex and full-duplex.

$0.90 \mathrm{~b} / \mathrm{s} / \mathrm{Hz}$ for full-duplex and half-duplex, respectively, and the gain factor due to full-duplex is 1.97. The corresponding RL average spectral efficiencies are shown in Fig. 6 as function of $\kappa$. The full-duplex average spectral efficiency increases with $\kappa$ and, at $\kappa=0.925$, it equals its half-duplex value; thereafter, the gain increases rapidly. Thus, full-duplex outperforms halfduplex only if the BSs are apart by at least the critical distance. This is indeed viable in microcell networks because of the relatively short critical distances (hundreds of meters).

\section{REFERENCES}

[1] M. Duarte and A. Sabharwal, "Full-duplex wireless communications using off-the-shelf radios: Feasibility and first results," in Proc. Annual Asilomar Conf. Signals, Syst., Comp., Nov. 2010, pp. 1558-1562.

[2] J. I. Choi, M. Jain, K. Srinivasan, P. Levis, and S. Katti, "Achieving single channel, full duplex wireless communication," in Proc. ACM int. conf. on Mobile comp. and net., Sept. 2010, pp. 1-12.

[3] M. Jain et al., "Practical, real-time, full duplex wireless," in ACM Int'l Conf. on Mobile Computing and Networking, Sept. 2011, pp. 301-312.

[4] D. Bharadia, E. McMilin, and S. Katti, "Full duplex radios," in Proc. ACM SIGCOMM, Aug. 2013, vol. 43, pp. 375-386.

[5] Z. Tong and M. Haenggi, "Throughput analysis for wireless networks with full-duplex radios," in Proc. IEEE Wireless Commun. and Networking Conf., Mar. 2015.

[6] C. Psomas and I. Krikidis, "Outage analysis of full-duplex architectures in cellular networks," in Proc. IEEE Veh. Technol. Conf., May 2015.

[7] S. Goyal, P. Liu, S. Hua, and S. Panwar, "Analyzing a full-duplex cellular system," in Proc. Conf. Inform. Sciences Syst., Mar. 2013.

[8] M. Haenggi, Stochastic Geometry for Wireless Networks, Cambridge Univ. Press, Cambridge, U. K., 2012.

[9] R. K. Mungara, D. Morales-Jiménez, and A. Lozano, "System-level performance of interference alignment," IEEE Trans. Wireless Commun., vol. 14, no. 2, pp. 1060-1070, Feb. 2015.

[10] J. Guo, S. Durrani, and X. Zhou, "Outage probability in arbitrarilyshaped finite wireless networks," IEEE Trans. Commun., vol. 62, no. 2, pp. 699-712, Feb. 2014.

[11] R. K. Mungara and A. Lozano, "Full-duplex MIMO cellular networks: System-level performance," preprint, 2015.

[12] 3GPP TR 36.828 V11.0.0, "Further enhancements to LTE Time Division Duplex (TDD) for Downlink-Uplink (DL-UL) interference management and traffic adaptation," Tech. Rep., 3rd Generation Partnership Project 3GPP, www.3gpp.org, 2012. 